\title{
Análisis de las implicaciones generadas por la miopía en la mercadotecnia empresarial
}

\author{
Izumi Sandoval, Taria Fabiola Andino Ruiz ${ }^{1}$ \\ Jesús David Argueta Moreno ${ }^{2}$
}

\section{RESUMEN}

La presente investigación exploratoria tiene como fin identificar las causalidades que inciden en el desempeño de ventas en el sector empresarial contemporáneo, generadas por el fenómeno conocido como "miopía en el mercadeo", el cual se describe como una anomalía que impregna todas las industrias, sin embargo, se acentúa en algunos rubros en particular, como el sector empresarial hotelero, del cual se describen algunos hitos en el análisis.

De igual forma, en esta investigación se exploran las implicaciones cualitativas que conllevan a la carencia del uso de habilidades investigativas adecuadas, absorbidas por el rubro empresarial a raíz de la falta de formación o preparación académica en algunos de sus empleados o colaboradores.

Palabras clave: cadena de valor y suministro, competitividad, sector empresarial, recursos humanos.

\footnotetext{
${ }^{1}$ Asistentes de investigación del Instituto de Investigaciones Económicas y Sociales, Facultad de Ciencias Económicas y Contables

${ }^{2}$ Asesor, Profesor universitario del Instituto de Investigaciones Económicas y Sociales, Facultad de Ciencias Económicas y Contables, UNAH: jargueta@iies-unah.org
} 


\section{ABSTRACT}

This research descriptive court saw fit to identify causalities that affect the sales performance of the business sector generated by the phenomenon known as " Myopia in Mercadeo ", which is a phenomenon that permeates the industry, but is accentuated particularly in some areas , one of which is the business sector, focusing on the hospitality industry.

Similarly, in this study the qualitative implications, created by the lack of in the field of vocational training, absorbed by the business area, as a result of lack of training / academic preparation in some of its employees / collaborators are explored.

Keywords: Supply Chain Management, Competitiveness, Business sector, Human Resources. 


\section{INTRODUCCIÓN}

El fenómeno de la miopía de mercadeo es hoy en día una anomalía a nivel global, la cual se manifiesta de manera anónima en las organizaciones, independientemente de su tamaño, rubro o longevidad. Distintos mercadólogos y expertos en la temática señalan que este fenómeno deriva de una diversidad de causalidades, cuya raíz emana de la carencia de protocolos de investigación bien definidos en las organizaciones (falta de burocratización de los roles y funciones), así como por la carencia de pericias, conocimientos y habilidades, de las cuales el personal en la organización debe de estar empoderado, a nivel de la investigación, desarrollo e innovación (I+D+i) (Kotler, Direccion en Marketing, 2009); asimismo, evaluando los componentes de las condiciones competitivas y macroambientales de la empresa, los cuales son factores decisivos en la aparición e intensidad de la miopía de mercado (Kotler, 2001).

Ante las secuelas de la miopía de mercadeo y, por ende, la mortalidad empresarial, la joven generación de profesionales se enfrenta con el reto de los nuevos y constantes cambios que la globalización y la innovación tecnológica imponen, por lo que las exigencias en su formación profesional son mayores, mismas que les permitirá posicionarse de una forma más ágil y estratégica en el mercado laboral local o internacional. Sin embargo, la realidad del contexto hondureño dificulta la rápida adquisición de un empleo y más aún, la inserción profesional en una instancia bien remunerada en el país (OML, 2011).

De manera paralela, el mercado laboral del sector empresarial, debido a las distintas condicionantes que afronta; a nivel de regulaciones tributarias, escases de proveedores, así como en su caudal de clientes, es imperativo que este se robustezca con el capital humano más apto para sus intereses financieros, comerciales y productivos (a nivel del I+D+i). Pero, en el sector privado latinoamericano, la tasa salarial promedio no es lo suficientemente atractiva para aquellos profesionales que cuentan con un grado académico o alguna certificación técnica en varios ámbitos profesionales, distorsionando así las potencialidades del recurso humano disponible para el rubro de las pequeñas y medianas empresas en América y de forma particular en Honduras (Kotler, Dirección de Mercadotecnia, 2001).

Lo expuesto con anterioridad lleva a la reflexión del porqué es importante para el fenómeno de la miopía de mercadeo evaluar las fricciones en el mercado laboral; asimismo, atender la interrogante del porqué muere una cantidad significativa de pequeñas y medianas empresas en América y de forma puntual en Honduras. Las 
cuales representan inquietudes que más allá de abordarlo con insumos de corte cuantitativo a nivel del volumen de creación, mortalidad, crecimiento y expansión empresarial en el medio, pues se deben abordar con insumos de corte cualitativo que impactan directamente en el rendimiento en ventas de las empresas y ayudan a conocer a plenitud las causalidades del nacimiento del fenómeno de la miopía de mercadeo, lo cual estimula la mortalidad empresarial, entre las cuales es importante mencionar:

1. Bajos niveles de educación a nivel media y superior que limitan la gestión del capital humano.

2. La tasa salarial promedio no es lo suficientemente atractiva para los profesionales que sí cuentan con una certificación o grado académico, vinculado al rubro empresarial.

3. La pequeña y mediana empresa recurre con frecuencia a estrategias de "upsizing" y "downsizing".

4. La tercerización en el ámbito empresarial se está volviendo un eslabón fundamental en la cadena de valor y suministro de sus servicios.

Cabe mencionar que para entender el funcionamiento de la relación actual entre la oferta y demanda de profesionales de la educación superior, media y técnica, contra el mercado laboral del sector empresarial, es imperativo entender el modelo de la cadena de valor y suministro del mercado laboral, concebido por el académico César Ortega, quien define cuatro ejes centrales: proveedor de ofertantes, ofertantes, demandante intermedio y demandante final, que se articulan entre sí y de manera cíclica atienden a los potenciales profesionales, según las distintas variantes y particularidades con las que cada eslabón cuenta (Ortega, 2012). A continuación se presenta el modelo de la cadena de valor y suministro de profesionales en la figura 1.

Finalmente, es de vital importancia mencionar que el fenómeno de la miopía del mercadeo depende de un sin número de causalidades de orden cuantitativo, así como cualitativo, sin embargo, en este estudio solamente se evaluarán a plenitud dos causalidades de orden cualitativo: distorsiones generadas por el mercadeo interno y las distorsiones implícitas en el sistema de negocios hondureños, exógeno a la pequeña y mediana empresa, generado por el mercadeo externo. 
Figura 1. Cadena de valor y suministro de la educación

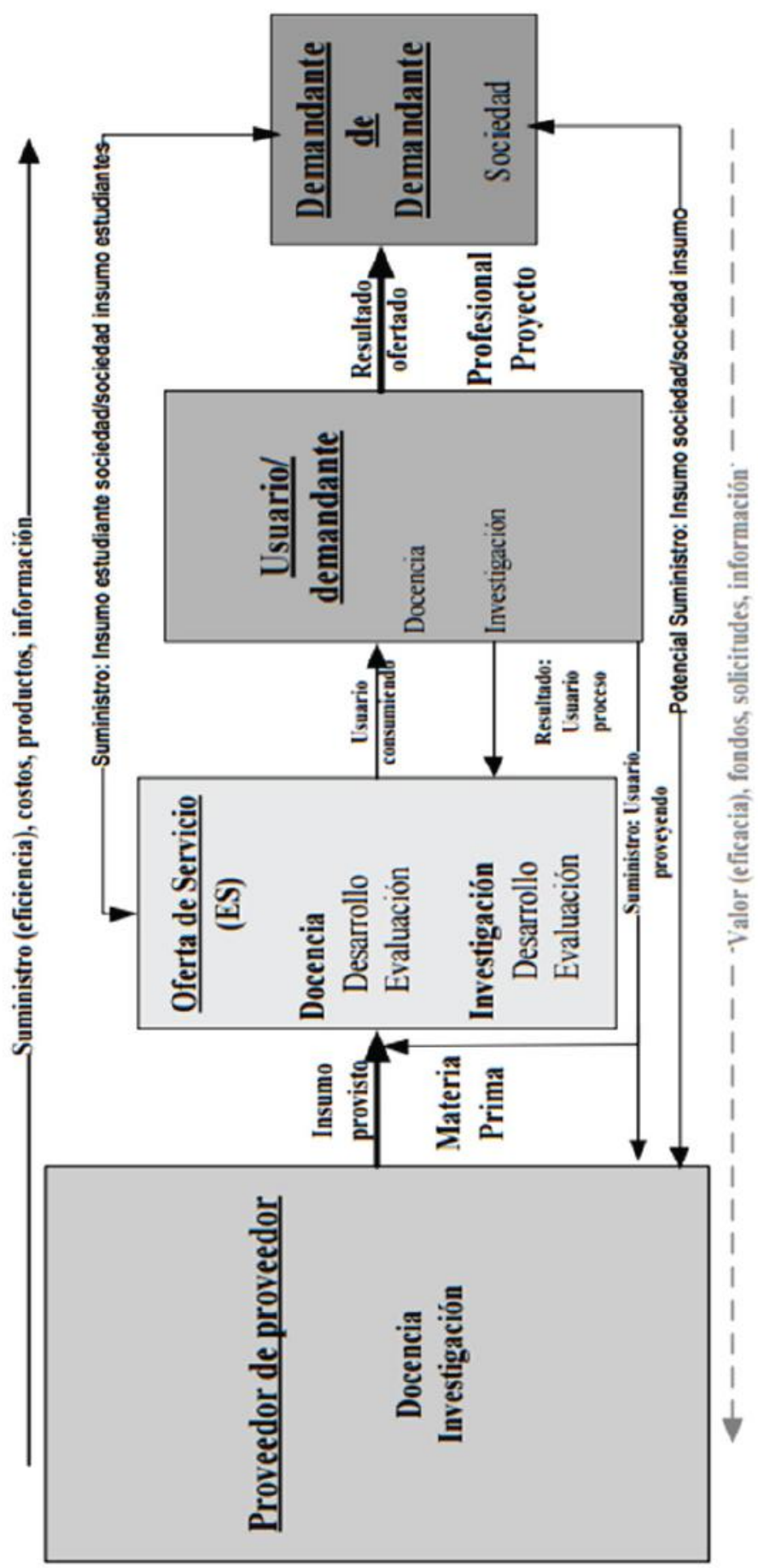

Fuente: Ortega, 2012 


\section{Antecedentes}

El fenómeno de la miopía de mercadeo se manifiesta en distintas intensidades y de forma particular en el rubro del turismo, en donde esta deformidad se acentúa con mayor notoriedad en el ámbito hotelero latinoamericano (Argueta, 2015). El turismo constituye uno de los servicios que por excelencia está generando más trabajos a lo largo de América Latina debido al crecimiento que ha experimentado en las últimas décadas. Esta industria, representa hoy en día una parte importante del producto interno bruto (PIB) de distintas naciones y, en algunas ocasiones, su margen de contribución es significantemente alto, como en el caso de la republica Dominicana $8.5 \%$, es por lo anterior que el concepto de competitividad no puede ser ajeno al rubro del turismo, al igual que los principios fundamentales de la calidad total y del mercadeo, que han descrito la anomalía de la miopía de mercadeo como una importante amenaza para la micro, pequeña y mediana empresa en el mundo y de forma particular en América Latina, en donde los factores especializados que garantizan la supervivencia de los hoteles se entrelazan y robustecen las fortalezas de la organización, de los cuales se describen algunos a continuación:

1. Recursos humanos.

2. Infraestructura diseñada para hacer accesibles los atractivos naturales (en los hoteles), mercados de capitales adecuados para financiar proyectos turísticos de largo plazo.

3. Niveles de seguridad personal adecuados y una alta cobertura de servicios públicos de apoyo que permitan valorar a plenitud todos estos atributos.

A nivel del sector turismo, América Latina, en especial América Central y el Caribe, han mostrado un aumento importante de participación en el mercado mundial en los últimos 3 quinquenios, lo que podría consolidarse en el futuro. Destacando que entre los años 2005 y 2014 estas regiones como Centroamérica y el Caribe han tenido un crecimiento anual del $4 \%$ y $2 \%$, respectivamente (Antón \& Rubio, 2006). Datos como el anterior, revelan la importancia de proteger la integridad financiera y competitiva de estas empresas, las que además de generar riqueza al país, producen conocimiento financiero, social y mercadológico, a través del I+D+i, que según varios especialistas tiene la virtud de contrarrestar o mitigar el fenómeno de la miopía de mercadeo (Puczkó, 2011). 
Cabe destacar que en el contexto latinoamericano, un fenómeno distorsionador de la planificación empresarial, así como de la estrategia organizacional, ha ido creciendo de forma desproporcionada, paralela al de la miopía de mercadeo, que es lo que se denominado "miopía administrativa", la cual tiene la capacidad de gestarse a nivel de todas las instituciones (públicas y privadas), con la particularidad de que sus atributos generan diversas falencias que disminuyen la capacidad de respuesta de las organizaciones mismas, al contar con información poco precisa y real sobre las condiciones productivas, comerciales y financieras de la empresa misma, sugiriendo medidas que están poco orientadas a las verdaderas falencias, debilidades y puntos de mejora en las empresas o instituciones en dificultades. De forma tal, que el espejismo facilitado por la miopía administrativa puede trasladarse a cualquier ámbito; de forma particular en este caso de evaluará la miopía en la rama del mercadeo, explicado por los expertos en gestión de proyectos en la terminología anglosajona como Project Scope Creep (Thakurta, 2013).

\section{Enunciados de la problemática}

En la actualidad, el concepto de miopía empresarial aún no se encuentra muy explorado por la academia y, en particular, una de sus tipologías como la miopía en el mercadeo, por lo tanto, en este documento se exploran las causalidades de esta anomalía, así como sus repercusiones en las ventas, eligiendo al sector hotelero para estos fines.

\section{Pregunta general de investigación:}

¿Existe evidencia empírica que describa la incidencia de la miopía en el mercadeo en el ámbito empresarial?

\section{Preguntas específicas de investigación:}

a. ¿Existen casos en Latinoamérica que documenten la existencia del fenómeno de miopía en el mercadeo en el ámbito empresarial?

b. ¿Existen casos a nivel mundial que documenten la existencia del fenómeno de miopía en el mercadeo en el ámbito empresarial?

c. ¿Cuáles son las causalidades que estimulan el desarrollo del fenómeno de miopía en el mercadeo? 


\section{Objetivo general de investigación:}

Conocer los casos de evidencia empírica que documentan la existencia y tratamiento de la miopía en el mercadeo del sector empresarial.

\section{Objetivos específicos de investigación:}

a. Documentar la evidencia empírica de los casos en Latinoamérica que registren la existencia del fenómeno de miopía en el mercadeo en el ámbito empresarial.

b. Documentar la evidencia empírica de los casos a nivel mundial que documenten la existencia del fenómeno de miopía en el mercadeo en el ámbito empresarial.

c. Determinar las causalidades que estimulan el desarrollo del fenómeno de miopía en el mercadeo en el sector empresarial hondureño.

\section{Justificación}

La detección de las condiciones y causalidades que estimulan la miopía en el mercadeo, sugiere al gerente de mercadeo contemporáneo la implementación de las mejores prácticas a nivel de la administración integral de proyectos, vertida por el Project Management Institute (PMI), las cuales se dividen en 47 procesos, para efecto de evitar, entre otras, los errores siquientes:

1. El consumo innecesario de recursos financieros de la empresa o institución en estrategias comerciales estériles.

2. El uso inadecuado de los recursos financieros, físicos y humanos de la empresa entorno a estrategias mal orientadas.

3. Poco o nulo conocimiento de la verdadera problemática que limita las condiciones de ventas de la empresa.

4. Formulación de estrategias comerciales erradas que condicionan la capacidad de respuesta de la organización o empresa.

Considerando que en los apartados anteriores se observa que es imperativo documentar la anomalía de la miopía en el mercadeo para tener un mejor entendimiento 
de esta y así evitar que continúe reproduciéndose a nivel del contexto hondureño y de forma particular en el ámbito empresarial.

\section{FUNDAMENTACIÓN TEÓRICA}

En este apartado se expondrán los conceptos ligados a la anomalía denominada miopía de mercadeo, denotando un marco conceptual de las temáticas en estudio, para luego proceder a tipificar la evidencia empírica de algunos estudios de casos atinentes al fenómeno de miopía de mercadeo a nivel global, de América Latina, América Central y el Caribe, a través de un marco referencial de la teoría.

\section{Marco conceptual}

En este componente se expondrán los conceptos relacionados con la temática de la miopía de mercadeo que apoyan el entendimiento de sus orígenes, causalidades y consecuencias. Para efecto de describir de manera integral el concepto, se utilizará la metodología de la administración de proyectos, al descomponer estos dos apartados, describiendo en primera instancia el concepto de mercadeo, sus dimensiones, etimología, evolución y procesos. De igual forma, se describirá el concepto de miopía, para luego articular ambos elementos en el concepto de miopía de mercadeo (PMI, 2013).

Al adentrarse en la temática de mercadeo, es vital mencionar que varios estudiosos como Philip Kotler (2001), Jerome McCarthy (1960), John Howard (1993) y Jack Trout (2006), entre otros, han intentado consensuar una definición universal para esta disciplina y, sin lugar a dudas, la definición de mercadeo con más aceptación entre los teóricos, mercadólogos y especialistas en la temática menciona lo siguiente: "La mercadotecnia es un proceso social y administrativo mediante el cual grupos e individuos obtienen lo que necesitan y desean a través de generar, ofrecer e intercambiar productos de valor con sus semejantes" (Kotler, 2001).

a. Evolución del mercadeo: el término mercadeo apareció en la literatura económica norteamericana a principios del siglo pasado, aunque con un significado distinto del actual. En Latinoamérica se suele traducir como mercadología, mercadeo, comercialización o mercadotecnia. Pero, ninguno de los términos anteriores expresa todo el contenido de la palabra inglesa; por el contrario, tienden a limitar su significado y objeto de estudio; razón por la cual se contempló 
utilizar estrictamente la palabra mercadeo, la cual goza de un amplio reconocimiento internacional, siendo utilizada ampliamente en el campo académico y profesional y reconocida por la Real Academia Española (Howard, 2002).

Se coincide con los mercadólogos Serrano, Santesmases y Martín Dávila, en que el mercadeo constituye una disciplina aún en desarrollo, la cual abarca un campo de conocimientos que se ha venido desarrollando, pero que todavía se encuentra en una etapa de desarrollo continuo en busca de su construcción y consolidación (Joseph Cannon, E. Jerome McCarthy , Jr. Perreault, William, 2003).

Al compilar la literatura relacionada al mercadeo, varios teóricos coinciden Coca y Milton (2008), en que existen tres períodos relevantes para categorizar el desarrollo del mercadeo como una disciplina: preconceptual (1900-1959), de conceptualización formal (1960-1989) y período actual (1990-a la fecha).

1) Periodo preconceptual: a inicios del período preconceptual nacen las primeras aportaciones acerca del concepto mercadeo, concibiéndose como parte de la economía que pone en contacto la producción con el consumo, estableciendo una relación directa en oferta y demanda. A esta función de comercializar los productos se le agrega la distribución física de los mismos en la década 1920, permaneciendo de esta forma hasta la mitad del siglo pasado, cuando los investigadores empezaron a percibir que el objeto de estudio de la disciplina contaba con un alcance mucho más extenso.

2) Periodo de la conceptualización formal: en 1960 se crea la Asociación Americana de Mercadeo (AMA), la cual estaba obligada a introducir la primera definición formal de la disciplina, aceptada mayoritariamente por la comunidad científica internacional. No obstante, en 1965, el Mercadeo Staff of the Ohio State University introduce la consideración del mercadeo como un proceso social; a partir de esta definición surge el núcleo de las discusiones alrededor del intercambio activo de valor, emergiendo el mismo como base del concepto. Como resultado se percibe una ampliación del alcance del mercadeo en dos sentidos: una ampliación vertical y una horizontal. La primera relacionada con la responsabilidad social del mercadeo, estableciéndose un orden ético jerárquico importante; la segunda relacionada con el despliegue del mercadeo desde las organizaciones estructuralmente lucrativas hacia las no lucrativas, del que surgiría el mercadeo social, cuyos impulsores más importantes serían Kotler y Levy (1969). 
3) Periodo actual: posteriormente a principios de los ochenta, se generan dos corrientes de pensamiento entorno al alcance del concepto de mercadeo: la norteamericana y la nórdica, que son las pioneras en la elaboración de una teoría del mercadeo de servicios, demostrando que la visión tradicional no se adapta a las particularidades de los productos intangibles. Serán estas también las encargadas de incorporar al intercambio una dimensión temporal no contemplado hasta el momento, propugnando una visión continua del mismo más cercana a la relación, frente a la actual visión desde un punto de vista discreto y puntual. Asimismo, en esta década se produce la adecuación del mercadeo hacia el enfoque estratégico, lo que se puede observar en la definición de la AMA (1985), en donde se introduce la necesidad de la proactividad con el entorno. Finalmente, en el año 2006, los especialistas en mercadología Kotler y Keller proponen un nuevo concepto de mercadeo, el cual describe a la disciplina más aceptadamente en la actualidad. Según los autores, desde el punto de vista de los negocios, mercadeo es el proceso de planear y ejecutar el concepto, el precio, la promoción y la distribución de ideas, bienes y servicios con el fin de crear intercambios que satisfagan los objetivos particulares y de las organizaciones (Kotler, 2009).

b. El mercadeo y sus dimensiones: si bien el término de mercadeo es por excelencia una herramienta indispensable en la elaboración de estudios de pre y factibilidad, así como en el diagnóstico de gestión para cualquier iniciativa de proyecto, este concepto se refiere a la reducción de la incertidumbre en torno a las eventualidades latentes y manifiestas, las cuales se pueden mitigar, evitar, transferir o aceptar, dependiendo del análisis de la magnitud de fenómeno 0 riesgo, restando así empirismo en el manejo de la información en las empresas u organización, igualmente en las estrategias que de esos insumos emana (Kotler, 2009). Por lo anterior, es importante mencionar que para atacar la gestión del riesgo se formularon 2 tipologías generales de mercadeo, las cuales cuentan con varias subcategorizaciones que les posibilitan mediar a eventualidades más puntuales (ver tabla 1 ). 
Tabla 1. Dimensiones o tipologías del mercadeo según la AMA

\begin{tabular}{|c|c|c|}
\hline $\begin{array}{l}\text { Dimensiones generales del } \\
\text { mercadeo }\end{array}$ & $\begin{array}{l}\text { Su dimensiones del } \\
\text { mercadeo }\end{array}$ & Protocolos del mercadeo \\
\hline \multirow{3}{*}{ Mercadeo interno } & $\begin{array}{l}\text { Recursos humanos de la } \\
\text { empresa }\end{array}$ & \\
\hline & Capacidad de la empresa & \\
\hline & Cultura de la empresa & \\
\hline \multirow{29}{*}{ Mercadeo externo } & \multirow{4}{*}{$\begin{array}{l}\text { Mercadeo de aspectos } \\
\text { legales }\end{array}$} & $\begin{array}{l}\text { Evaluación } \quad \text { cumplimiento } \\
\text { normas ISO }\end{array}$ \\
\hline & & Evaluación leyes antitrust \\
\hline & & Evaluación leyes fiscales \\
\hline & & Evaluación tarifas bancarias \\
\hline & \multirow[b]{5}{*}{ Mercadeo ecológico } & Protección ambiental \\
\hline & & Eliminación de residuos \\
\hline & & Fiabilidad de los productos \\
\hline & & Reciclaje productos \\
\hline & & Conservación del entorno \\
\hline & \multirow[b]{6}{*}{ Mercadeo tecnológico } & Innovaciones \\
\hline & & Nuevos productos \\
\hline & & Aumento de la productividad \\
\hline & & Legislación de patentes \\
\hline & & Aplicaciones robóticas \\
\hline & & Desarrollos informáticos \\
\hline & \multirow{6}{*}{ Mercadeo económico } & Tasas de interés \\
\hline & & Oferta monetaria \\
\hline & & Tasa de inflación \\
\hline & & Niveles de empleo \\
\hline & & Aumento del PNB \\
\hline & & $\begin{array}{l}\text { Costo de los factores de } \\
\text { producción }\end{array}$ \\
\hline & \multirow{8}{*}{ Mercadeo competitivos } & No competidores \\
\hline & & Tamaño de los competidores \\
\hline & & Crecimiento del mercado \\
\hline & & Participación del mercado \\
\hline & & Políticas de precios \\
\hline & & Ingreso en la industria \\
\hline & & Competencia internacional \\
\hline & & Políticas de productos \\
\hline
\end{tabular}

Fuente: Interpretación de las categorizaciones del mercadeo contemporáneo según la AMA, 2015. 
c. La miopía empresarial: entendiendo que el concepto de miopía hace alusión a la dificultad de vislumbrar un escenario determinado, la forma en la cual se utilizó el concepto de miopía en este análisis parte de la premisa empresarial, la cual contempla la toma errada de datos que más hayan de ser desviados, sesgan su interpretación al intentar atacar los riesgos que se reproducen en varias iniciativas de proyectos empresariales con información limitada y poco precisa sobre los fenómenos (Levitt, 1960). Cabe mencionar que es precisamente el modelo del iceberg, el cual explica y simula de mejor manera este fenómeno, en donde el amplio desconocimiento de la problemática condiciona el actuar de la empresa, impidiendo que se ataquen las causalidades del mismo, a nivel integral. Situación en la cual se cuantifica o analiza las dificultades de manera superficial, sin ahondar en las raíces del riesgo (Kotler, 2001).

Por lo descrito anteriormente, se asevera que al evaluar de manera conceptual el termino de miopía, bajo el marco administrativo y de manera particular el nivel de la herramienta de mercadeo, es vital señalar que el acercamiento teórico más acentuado es el propuesto por el académico Theodore Levitt a mediados de la década de 1960, quien menciona sobre la existencia del paradigma que todas las empresas nacen con la pretensión de vender un buen producto al cliente y que la clave era producir un producto de calidad, lo que les aseguraba la preferencia del consumidor ante la competencia. Sin embargo, enmarcados poco predecibles, más volátiles, es primordial que alguien se dé a la tarea de responder lo siguiente: ¿Muestra interés el personal de la empresa en detectar si el producto ofertado es realmente lo que el cliente necesita? ¿O la empresa simplemente había explotado un "know-how" (conocimientos o experiencia) y había ofrecido al cliente lo que mejor sabía hacer? ¿Qué papel tenía el cliente? Estas interrogantes son oportunas con el afán de optimizar el uso de las herramientas de mercadeo y eventualmente aumentar la capacidad de respuesta de las empresas que le utilizan (Levitt, 1960).

De igual forma, el académico Levitt, señala, que la Miopía en el mercadeo, se exhibe en la mayor parte de los casos, como una forma de desconocimiento exógeno de la empresa, de forma puntual a nivel de las siguientes categorías: Mercadeo de Aspectos Legales, Mercadeo Tecnológico, Mercadeo Económico, entre otras. las cuales pertenecen a la tipología de Mercadeo Externo, reproduciéndose aquí el teorema de Wilfredo Paretto, a nivel que el $20 \%$ de los principales problemas son de corte exógeno, sin embargo alteran el $80 \%$ de las gestiones estratégicas de la empresa (Levitt, 1960). 


\section{MARCO REFERENCIAL}

En este apartado se tipificarán algunos casos documentados en los cuales la miopía de mercadeo se manifestó, detecto y gestiono, a nivel global, regional y local.

\section{Miopía de mercadeo a nivel global}

\section{a. El caso Kodak}

Kodak es uno de los casos más conocidos, dado que su miopía fue notable, ya que sus factores de fracaso pudieron ser superados con el mercadeo interno, sin embargo, dejaron que el mercadeo externo los superara en tecnología, desarrollo informático, competidores, crecimiento del mercado, políticas de producto; estos fueron algunos de los factores que hicieron que una empresa tan prestigiosa como la Kodak quedara fuera del mercado.

La Eastman Kodak Company fue fundada en 1888, inició con la introducción del carrete de papel en el mercado, reemplazando las placas de cristal que se empleaban hasta entonces, fue una de las primeras cámaras que empleaban carretes circulares. Su lema describe bien su propuesta: "Usted aprieta el botón, nosotros hacemos el resto". Fueron los primeros en crear fotos a todo color y en crear un producto para hacer fotos incluso debajo de agua (Gonzales, 2012).

Desde su aparición, Kodak lideró el desarrollo comercial de la fotografía, gracias a innovaciones tecnológicas que gozaron de ciclos de vida extensos. Tuvo durante 130 años una posición envidiable en el mercado, vendían el $85 \%$ de las cámaras fotográficas en todo el mundo y el $90 \%$ de las películas a nivel global. Supieron crear un modelo de negocio totalmente rentable gracias al bajo precio de la cámara (Ochoa, 2015).

La misión de Kodak era: "Proveer a sus consumidores con las soluciones necesarias para capturar, almacenar y comunicar imágenes donde sea y cuando sea", pero con una manera conservadora de mantener su negocio ante la irrupción de las nuevas tecnológicas, dejaron que la tecnología los absorbiera, ya que la resistencia al cambio es el fracaso de muchas empresas que creen que seguirán siendo líderes sin hacer el cambio que los consumidores exigen (Bernal, 2012). 


\section{b. Caso Grupo Océano}

El grupo Océano jugo un papel importante en el desarrollo y apoyo de la educación en muchas escuelas, colegios y universidades, fue una herramienta. Pero, una de sus estrategias fallidas fue el mercadeo externo del desarrollo informático, porque los libros fueron quedándose rezagados; por lo tanto, surgieron problemas con el nivel de empleo al cerrar muchas librerías, debido al aumento de los libros digitales, el crecimiento del mercado, participación del mercado, el mercadeo interno y la cultura de la empresa, ya que ellos querían conservar las librerías tradicionales.

Grupo Océano es un grupo empresarial español dedicado a la edición y distribución de contenido literario en niveles de enciclopedias y manuales prácticos, fundado en 1959 por José Luis Monreal. Tiene agencias en 21 países de habla española, además de Portugal, Brasil y Estados Unidos, se convirtieron en un apoyo laboral para el docente, referencia de muchos estudiantes y una oportunidad para aprender idiomas, teniendo mayor impacto en América Latina, especialmente en América Central en donde poseían una agencia de distribución en cada país (Branson, 2015).

Las populares enciclopedias tuvieran éxito con los años; en 1972 adquieren la editorial Éxito, teniendo auge en 1983. Con el avance del internet, en el año 1994, los niveles de ventas comienzan un declive, de modo que en 2002, se crea una nueva Océano digital, con la cual se introducen en la literatura digital, con poco impacto sobre su mercado (Branson, 2015). Por último, se un esfuerzo para mantenerse en el mercado y es cuando en 2011 nace Océano Link, ofreciendo una línea editorial para obras de arquitectura y diseño, ya que el diseño y la publicidad se vuelve una tendencia en la oferta académica superior, aunque tampoco resulta ser un líder en ese mercado, ya que hay programas especiales como Corel, Adobe y Photo Shop con licencias que incluyen varios tutoriales. Océano cerró operaciones en el año 2014 (Swan, 2014).

\section{c. Caso Borders Book}

Borders Book se escogió como un caso de estudio, ya que fue una de las marcas más impotentes en los Estados Unidos; teniendo un alto porcentaje de participación, las librerías intentaron sobrevivir, sin embargo, el recurso personal fue uno de los perjudicados, debido a sus sucursales cerradas, mantener una cultura empresarial, con problemas con la evaluación con tarifas bancarias; asimismo, 
porque pidió un préstamo que se le dificulta pagar, teniendo competencia internacional, y pierden la participación del mercado.

Borders Group fue un minorista de libros y música fundada en 1971 por los hermanos Tom y Louis Borders, esto comienza cuando estaban en la universidad, con sede en Ann Arbor, Michigan, Estados Unidos. Los hermanos Borders ofrecen sistemas de inventarios, su principal actividad era servir a los mayoristas y proporcionar los sistemas, (Pablo Oliviera y Silva , 2012).

Al presentar problemas financieros, en 2007, cuando se vendieron las acciones a un inversor de capital privado de Reino Unido, queda de nuevo solo con las tiendas de los Estados Unidos. En septiembre de 2009 suspenden el servicio inalámbrico basado en honorarios, proporcionada por T-Mobile, y comenzó a implementar una red wi-fi disponible por Verizon (Sanburn, 2011). Es cuando Borders realiza una alianza con Amazon para proporcionar la venta de libros, pero pocos libros son vendidos y el costo de mantenerlo en línea genera más gasto que utilidades. En el 2010 piden un préstamo para instalar una página web en donde se podían vender o rentar los libros; en 2011 un juez aprueba la petición de liquidación, cerrando las 399 tiendas, venden a su competidores (Barnes \& Noble) marcas, patentes y lista de clientes importantes, para poder pagas sueldos y préstamos que la empresa no pudo cubrir (Magee, 2011).

\section{Miopía de mercadeo a nivel regional}

a. Caso Taco Bell

Taco Bell es una de las empresa en la que se puede ver cómo por mantener su cultura empresarial con fallas, perdieron en algunos países su participación en el mercado, por no hacer una investigación de mercado adecuada, recurso humano, un incidente del cumplimiento de las normas ISO y la fiabilidad de sus productos. Tratando de mantener sus productos, realizan nuevos a base de diferentes ingredientes.

Glen Bell, en 1946, se puso a trabajar en un puesto de perros calientes, en Bell's Drive-In. En 1952, comenzó añadiendo productos alternativos a su menú, como los tacos; en 1962, dio un nuevo rumbo al negocio construyendo el primer Taco Bell en Downey y Kermit Becky, un expolicía de Los Ángeles, compró la primera franquicia de Taco Bell en 1964 (Bell, 2012). 
Bell, en 1978, vendió los 868 restaurantes a Pepsi Co Inc, con una visión de ofrecer comida de Taco Bell "en cualquier momento y lugar en que la gente tenga hambre". Su estrategia de ventas se baso en la instalación masiva de sucursales, próximas a centros de consumo masivo (aeropuertos, gasolineras, etc.), no obstante su fracaso en el contexto centroamericano responde a: La falta de investigación del mercado y por la falta de tropicalización/adaptabilidad, de los productos o servicios al mercado local (Torres, 2011). Esto es lo que ha pasado en varios países, ya que ellos quieren imponer su marca y su tradicional cocina estilo mexicano, sin cambios dirigidos a los consumidores y tradiciones del país en el que apertura.

Se ve en aprietos en 2010, porque en California fue fotografiado un empleado lamiendo una hilera de tortillas rígidas para tacos. En su defensa publicaron que las tortillas rígidas en forma de $U$, fueron suministradas a los empleados para practicar sus nuevos tacos Cool Ranch, aunque fueron botadas las tortillas y nunca se vendieron (Marketing, 2013)Aun con todas las dificultades, sigue teniendo participación en el mercado.

\section{b. Caso TACA}

TACA es un caso que no dejó de innovar, pero un accidente aéreo le quitó el prestigio y confianza en el mercado, por lo que es un caso de miopía, ya que las estrategias no resultaron, tuvieron una competencia internacional y se hizo una reducción de la participación del mercado, teniendo como consecuencia un nivel de empleo menor, ya que la economía de la empresa ya no era rentable.

En 1931 se funda Transportes Aéreos Centroamericanos (TACA) en Tegucigalpa, Honduras, como una empresa carguera, teniendo la prioridad de ofrecer a sus pasajeros una experiencia de vuelo caracterizada por la amabilidad, respeto y atención al cliente, cuya visión era: "Ser la aerolínea de preferencia del viajero moderno" y su misión: "Volar con calor, orgullo y pasión, en forma segura, eficiente, confiable y amable para nuestros clientes". En 2008, TACA renueva su imagen en forma integral, con el propósito de propiciar el alcance de su visión: ser la aerolínea preferida del viajero de las Américas; pero en julio del mismo año, TACA reportó el accidente de uno de sus aviones en el aeropuerto de Toncontín en Tegucigalpa, Honduras, vuelo que salió de San José, Costa Rica, en el que murieron varias personas y otras quedaron con severas secuelas (TACA, 2010). 
Teniendo consecuencias un declive en sus activos intagibles, a nivel de su marca,e imagen empresarial, desconfianza en la calidad profesional de sus funcionarios, disminuyen la cantidad de viajes, incredulidad en el sistema de mantenimiento correctivo y preventivo de sus aeronaves, dudas de la estabilidad económica, rumores de despido y en la flotilla de TACA. Todos estos factores permiten observar un análisis del ambiente industrial; el posicionamiento y marca se tornan negativos y entre la competencia se convierte en una de las aerolíneas débiles del mercado. Finalmente, en 2010, se hace la fusión con Avianca, ya que los accionistas concretan el cierre de la unión obteniendo las aprobaciones regulatorias, esta estrategia y cambio de imagen, marca, logo e imagen corporativa, lograron la confianza de los viajeros (Valdes, 2009).

\section{METODOLOGÍA}

La presente investigación se propuso develar las distintas causalidades por las cuales el riesgo latente de la miopía de mercadeo se materializa, afectando así la precisión de la información de gestión en las empresas contemporáneas y, por ende, sus estrategias comerciales y productivas.

La presente investigación es de tipo descriptiva, con la cual se tuvo que evidencia teórica y empírica sobre el fenómeno de la miopía de mercadeo, al describir su composición y formas de manifestación en el ámbito administrativo. En consecuencia, se hizo un diagrama de pescado, en el cual se plasman todas las inferencias sobre las casualidades que se detectaron en este análisis, al estudiar la reproducción de la miopía en el mercadeo de algunas empresas, a nivel global y regional.

Se construyó dicho diagrama en el marco de la compilación empírica (análisis de casos) y teórica (revisión de los manuscritos de Theodore Levitt).

\section{RESULTADOS}

Luego de recorrer el sustento teórico y empírico pertinente a la temática de la miopía del mercadeo, es válido inferir que la tipología que más se aplica es la del mercadeo externo y sus categorías, dentro de las cuales figura el mercadeo económico, tecnológico y competitivo. 
De forma paralela a la compilación del sustento teórico, tras la compilación de evidencia empírica, es válido inferir que esta anomalía altera las condiciones de capacidad de respuesta, adaptabilidad tecnológica, capacidad de innovar, sostenibilidad de las ofertas, congruencia mercadológica, capacidad de ajustar modelo de negocios y capacidad para anticiparse a riesgos de mercado.

Asimismo, es válido mencionar que en el marco de la temática de la miopía del mercadeo, el único modelo que describe plenamente su gestión, como un todo, es el modelo formulado a mediados de los años 1960 por Theodore Levitt, quien la define como un riesgo aún latente que puede ser mitigado, transferido, aceptado o evitado. Finalmente, es importante mencionar que tras recabar distinta evidencia empírica a nivel global y regional, se desarrolló un análisis de causa y efecto para mapear a las distintas causalidades que dan forma al alcance del fenómeno de la miopía de mercadeo, enmarcado en las dimensiones del concepto del mercadeo como tal (ver figura 2).

El caso de la miopía del mercadeo es importante señalar que las empresas en análisis, formularon estrategias a largo plazo, las que al final se volvieron metas de corto plazo. No obstante, ya que estas instituciones omitieron los pasos para el desarrollo de un efectivo mercadeo interno y externo, fueron organizaciones que se aferraron a corrientes de pensamiento tradicionales.

El error es tener la percepción de que al ser rentables, y quizá líderes de su sector, pueden mantenerse en una "zona de confort permanente", la cual, sin lugar a dudas, limitó para que se ajustaran a las nuevas tendencias, sin investigar y modificar su producto, solo basándose en las necesidad de los diferentes mercados. Estas empresas deben ser siempre innovadoras, tratar de ser las más astutas, para seguir siendo lo mejor que tiene el rubro; el desarrollo de productos u optimizar los ya existentes, ayuda a que los consumidores tengan muy presente el bien o servicio ofertado. 
Figura 2. Interpretación de las principales causalidades del fenómeno miopía del mercadeo

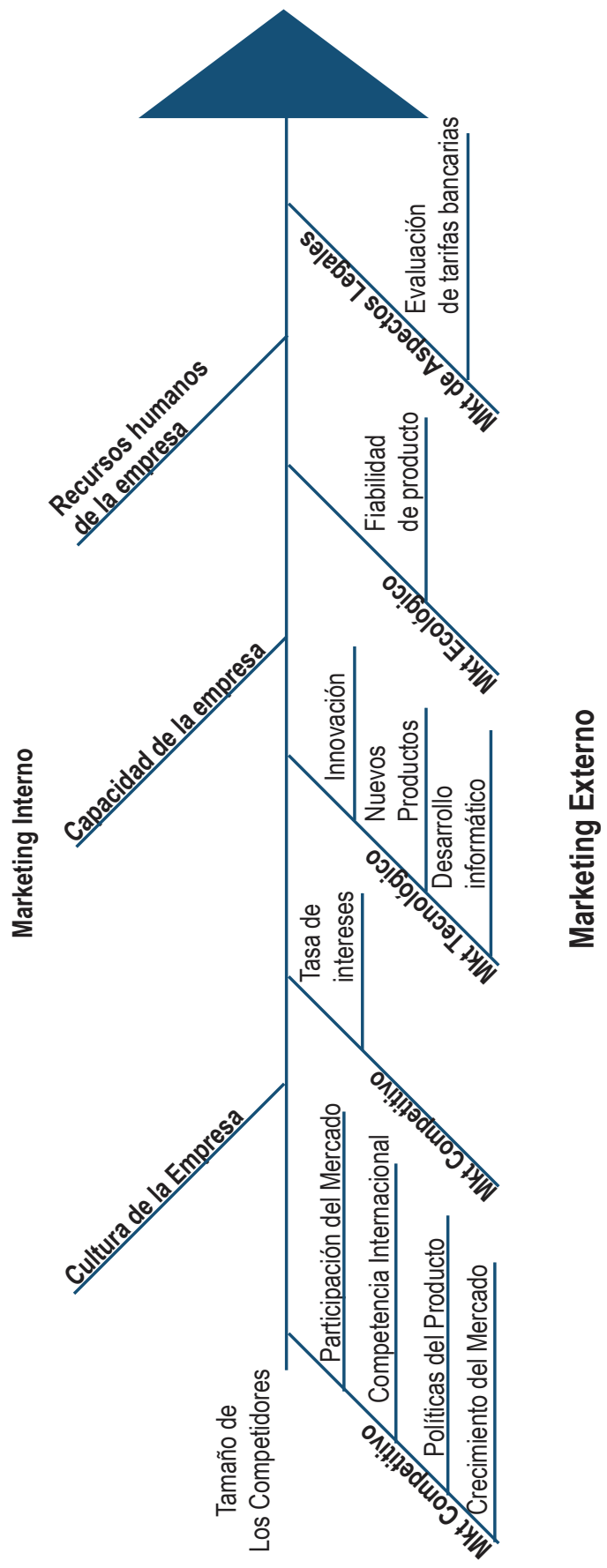

Fuente: Intepretación personal de los factores que inciden en la miopía del mercadeo, basado en evidencia empírica recabada sobre el fenómeno. 


\section{CONCLUSIONES}

1. En América Latina, la miopía del mercadeo suele parecer insignificante, no obstante, este fenómeno ha demostrado ser capaz de estimular riesgos con enorme potencial de ocurrencia y de deterioro hacia los activos de las organizaciones, en el caso de que no se consiga detectar en su fase latente; por consiguiente, es imperativo que las instituciones en América Latina sensibilicen a sus asociados entorno al tratamiento de esta temática.

2. Independientemente de que las empresas cuenten con una posición ventajosa en el mercado o que estas sean muy rentables, la cultura de calidad, innovación y mejora continua es dinámica y, por tanto, no debe detenerse o frenarse por ningún motivo. Esta situación llama a las empresas contemporáneas a que no se estanquen en su zona de comodidad y continúen estimulando la investigación, desarrollo e innovación (l+D+i).

3. Las causalidades más frecuentes de la miopía del mercadeo que se observaron en el estudio, parten de la dimensión de mercadeo externa, considerando aspectos legales y económicos debido a las tarifas bancarias que las empresas no pueden pagar. Ante tal situación, no invierten en nuevas líneas de productos, cuidando la fiabilidad de la procedencia de su materia prima en el desarrollo del producto o servicio.

4. Finalmente, al analizar los casos a nivel empresarial con énfasis en el fenómeno de la miopía del mercadeo, se observa que simplemente basaban sus estrategias empresariales en el mantenimiento de una cultura y políticas mercadológicas (visión y misión) integrales, sin ahondar en elementos investigativos de calidad que les podrían ayudar a comprender de una mejor manera los cambios del entorno y el comportamiento de los consumidores finales, de manera más rápida y dinámica.

\section{AGRADECIMIENTO}

A la Dirección de Investigación Científica y de Posgrados de la UNAH, por permitirnos publicar este artículo para compartir nuestros trabajos de investigación. 


\section{BIBLIOGRAFÍA}

Antón, J., \& Rubio, L. (2006). PROPUESTA DE CREACIÓN DE UN MODELO DE CAPITAL INTELECTUAL HOTELERO LATINOAMERICANO. Estudios Turísticos, n.o 167 (2006), 55-90.

Argueta, J. (2015). Como Afecta el Alto Empirismo en la Cadena de Valor y Suministro del Servicio Hotelero en Honduras. https://dicyp.unah.edu.hn/eventos/9no-congreso-de-investigacion-cientifica-unah-2015/ (1-12). Tegucigalpa : Investigación Congreso DICYP 2015.

Bell, T. (3 de Mayo de 2012). Historia de Taco Bell. Obtenido de Taco Bell : http://www.tacobell.es/category/historia/

Bernal, C. M. (2012). Caso Kodak Análisis de la o las fallas cometidas por Kodak en

su gestión y las estrategias tomadas o soslayadas, que llevaron a la empresa a la actual situación. Barranquilla, Atlántico, Colombia. CM Consultores .

Gonzales, C. (2012). El Curioso Caso de Kodak. Historia de Emprendedores. Recuperado de: http://www.negocios1000.com/2012/05/el-curioso-caso-del-fracaso-de-kodak.html

Howard, J. (2002). Pensamiento del Marketing Moderno. México.D.F. Pearson Educacion .

Joseph Cannon, E. Jerome McCarthy , Jr. Perreault, William. (2003). Comercialización básica. En Elementos esenciales de marketing (92-95). México.D.F. Pearson educacion.

Kotler, P. (2001). Dirección de Mercadotecnia. Lima, Peru: Pearson Education.

Kotler, P. (2009). Direccion en Marketing. En P. Kotler, Direccion en Marketing . Madrid. Pearson Prentice hall .

Levitt, T. (1960). Marketing myopia. Harvard Business Review, Edicion julio-agosto. 45-56.

Magee, D. (22 de Julio de 2011). International Bussiness Times . Obtenido de International Bussiness Times : http://www.ibtimes.com/borders-closing-why-bookstore-chain-failed-300009

Marketing, A. (6 de Mayo de 2013). La Voz. Obtenido de Taco Bell echa a empleado que fue fotografiado lamiendo tortillas: http://www.lavoz.com.ar/ciudadanos/taco-bell-echa-empleado-que-fue-fotografiado-lamiendo-tortillas

Ochoa, C. (5 de Enero de 2015). EL CASO KODAK, MUCHO QUE APRENDER. Obtenido de Blog la actualidad sobre la investigacion por internet : http://www.netquest.com/blog/es/el-caso-kodak/

OML. (2011). Reporte Anual Observatorio Mercado Laboral. Tegucigalpa, Francisco Morazan, Honduras: Portal de Transparencia Gobierno de Unidad Nacional. 
Ortega, C. (2012). Cadena de valor de la vinculación universidad-demanda de educaciónsuperior y las cadenas de suministro de docencia e investigación: Eficacia y eficiencia en la educación superior. XIII Asamblea General de ALAFEC, 88-116.

Pablo Oliviera y Silva . (2012). Borders la egunda libreria mas importante de los Estados Unidos. Marketing Actual .

Pepper, A. Z. (15 de Julio de 2011). Caso TACA Airlines. Obtenido de Prezi : https://prezi.com/7axw51vmrprj/caso-taca/

PMI. (2013). 5ta Edición Guia de Administración de Proyectos. Mexico: Mc Graw Hill. Puczkó, J. (2011). Wellness tourism and medical tourism : where do spas fit? New York: Global Spa Summit; 2011, http://bit.ly/1JzS6o.

Sanburn, J. (19 de Julio de 2011). 5 Razones por las que Borders Salio del Mercado. Obtenido de TIME : http://business.time.com/2011/07/19/5-reasons-borders-went-out-of-business-and-what-will-take-its-place/

TACA. (2010). Historia de TACA Airlines. Obtenido de Empleadosavianca.com : http://www.empleosavianca.com/aboutus.aspx

Thakurta, R. (2013). Impact of Scope Creep on Software Project Quality. Vilakshan, XIMB Journa, Vol.10 (1) .

Torres, J. (27 de Mayo de 2011). Marcas que fracasaron en Peru. Obtenido de Blanding UPC: http://mantramkt.blogspot.com/2011/05/fracaso-de-maca-caso-5-taco-bell-en.html

Valdes, V. H. (Octubre de 2009). Regulación económica de aerolíneas en el Istmo Centroamericano. Obtenido de cepal.org: http://www.cepal.org/es/publicaciones/4891-regulacion-economica-de-aerolineas-en-el-istmo-centroamericano 